\title{
Development Status of the International Space Station Urine Processor Assembly
}

\author{
Donald W. Holder and Cindy F. Hutchens \\ NASA, Marshall Space Flight Center
}

Copyright $\odot 2003$ Society of Automotive Engineers, Inc.

\begin{abstract}
NASA, Marshall Space Flight Center (MSFC) is developing a Urine Processor Assembly (UPA) for the International Space Station (ISS). The UPA uses Vapor Compression Distillation (VCD) technology to reclaim water from pre-treated urine. This water is further processed by the Water Processor Assembly (WPA) to potable quality standards for use on the ISS. NASA has developed this technology over the last 25-30 years. Over this history, many technical issues were solved with thousands of hours of ground testing that demonstrate the ability of the UPA technology to reclaim water from urine. In recent years, NASA MSFC has been responsible for taking the UPA technology to "flight design" maturity. This paper will give a brief overview of the UPA design and a status of the major design and development efforts completed recently to mature the UPA to a flight level.
\end{abstract}

\section{INTRODUCTION}

The UPA uses vapor compression distillation technology to process urine. This technology has been in development for approximately 30 years. During that time, many system design and operational issues have been identified and fixed through a long series of analyses and tests (both at the component and system level). By the time the VCD technology was selected to be the baselined urine processing technology for the ISS U.S Segment and the detailed design and manufacturing for the flight system was kicked off in the late 1990's, most of the major technology issues had been addressed.

However, in laying out the final, detailed design and defining the flight requirements (performance, environmental and safety) that the UPA must meet, it became apparent that there was still development work to be done in some key areas for the UPA to operate safely in microgravity and still process urine. This paper will address the 4 major areas of system development and design validation since the UPA program was started in 1998. Specifically, these areas are: 1) Condensate Collection in the Stationary Bowl 2) Acoustics and Microgravity Compliance 3) Microgravity Performance Validation, and 4) Compatibility with Processing Russian Pre-treated Urine. As with any flight program, issues have also risen relative to component design and manufacturing. A summary of the major component design and manufacturing issues and a manufacturing status of the UPA flight hardware is described elsewhere (see reference 1 ).

\section{URINE PROCESSOR ASSEMBLY OVERVIEW}

A simplified schematic of the UPA is shown in Figure 1. Urine is delivered to the UPA either from the Node 3 Waste and Hygiene Compartment (currently scarred only) or it can be supplied via manual transfer from the Russian EDV. The urine is temporarily stored in the Wastewater Storage Tank Assembly (WSTA) until it reaches a setpoint to begin processing. The Fluids Control and Pump Assembly (FCPA) is a four-tube peristaltic pump which moves urine into the Distillation Assembly (DA), concentrated waste from the DA into the Recycle Filter Tank Assembly (RFTA), and product water to the interface with the WPA. The DA is the heart of the UPA, and consists of a rotating centrifuge where water is evaporated from the waste urine stream at very low pressure. A rotary lobe compressor provides the driving force for the evaporation and compression of water vapor. Waste brine resulting from the distillation process is concentrated in the RFTA. The Pressure Control and Pump Assembly (PCPA) is another fourtube peristaltic pump, which removes non-condensable gases and water vapor from the DA. These gases are pumped to the Separator Plumbing Assembly (SPA), which recovers and returns water from the purge gases to the product water stream. A Firmware Controller Assembly (FCA) provides the command control, excitation, monitoring, and data downlink for UPA sensors and effectors.

The UPA is designed to process a nominal load of 8.45 $\mathrm{kg} /$ day (18.6 lbs/day) of wastewater consisting of urine, flush water, and a small amount of waste from 
Environmental Health System water samples. At a maximum load, the UPA can process $13.6 \mathrm{~kg}$ ( $30 \mathrm{lbs}$.) of wastewater over an 18-hour period per day. It operates in a batch mode, consuming $424 \mathrm{~W}$ power when processing, and $108 \mathrm{~W}$ during standby (current projections). Product water from the UPA must meet specification quality requirements for conductivity, $\mathrm{pH}$, ammonia, particles, and total organic carbon. It must recover a minimum of $85 \%$ of the water content in the specified wastewater stream.

The UPA is packaged into 7 ORUs, which take up slightly more than half of the WRS Rack \#2. The RFTA is the only expendable ORU, designed for a 30-day changeout.

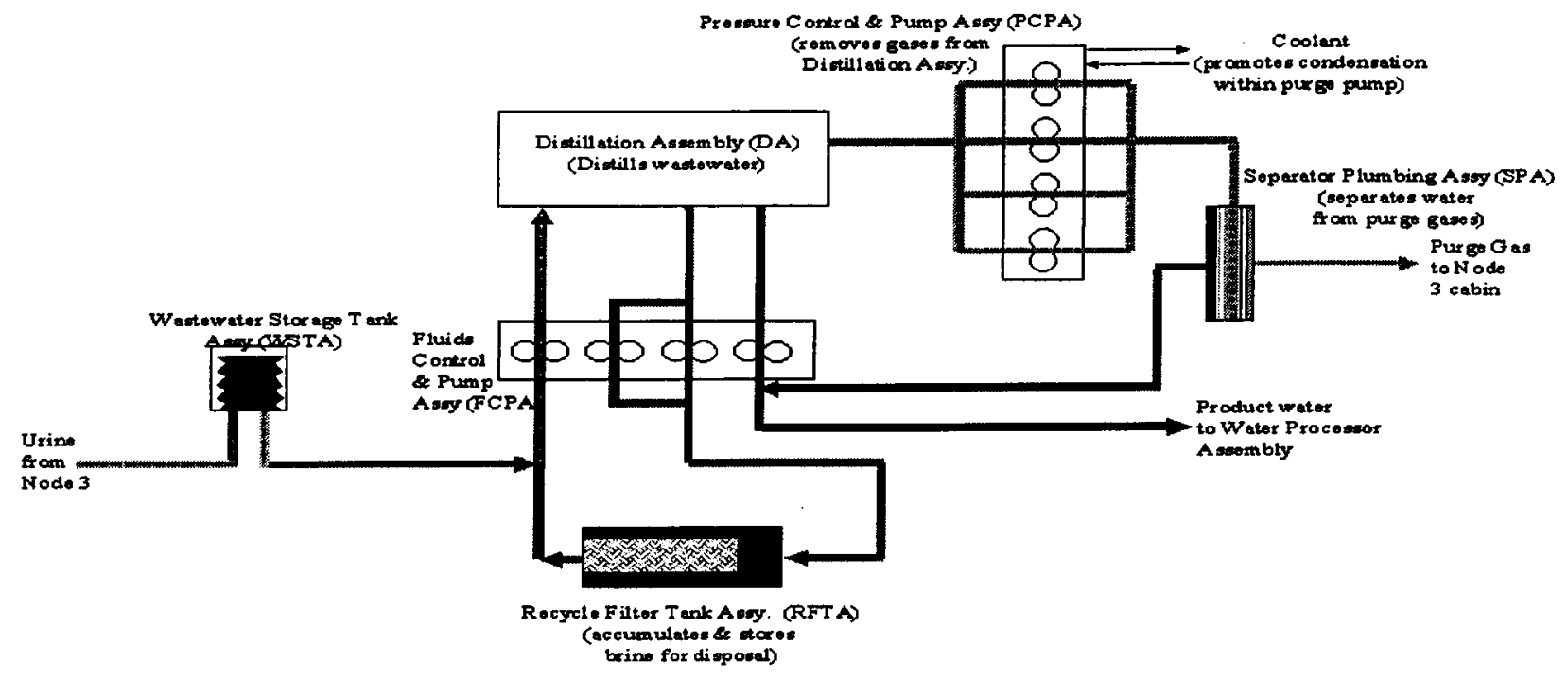

Figure 1. Urine Processor Assembly Simplified Schematic

\section{UPA SYSTEM DEVELOPMENT STATUS}

\section{CONDENSATE COLLECTION IN THE STATIONARY}

BOWL - In order to understand this issue, it is necessary to understand the operation of the Distillation Assembly. As stated previously, urine is pumped into the DA from the FCPA. It enters the rotating evaporator through the feed tube (Figure 2). The urine is spread onto the evaporator wall in a thin film that travels the length of the evaporator until it is collected in the urine/brine trough and pumped out by the FCPA through the evaporator pickup tube. As it travels down the wall of the evaporator, water is evaporated from the urine. The steam that is generated is pumped through the center of the hollow stationary shaft and compressed in the condenser by the compressor. Once the steam's pressure is raised, it begins to condense and give up its latent heat to the evaporator. This latent heat plus the waste heat generated from the compressor and motor are what provide the energy for the evaporation of water from the urine. The steam then condenses and, due to centrifugal force, collects on the outer wall of the condenser and travels to the product water trough. There it is pumped out of the condenser by the FCPA through the product water pickup tube. This entire process is done under vacuum. The vacuum is contained by the stationary bowl which surrounds the rotating still and compressor.

The 2-phase operation is controlled in microgravity by the centrifugal force of the rotating still. This allows the $D A$ to control the location of liquid and steam in the DA so that the evaporation and condensation process can take place and the liquids can be pumped into and removed from the DA. However, it was discovered that because there is no seal between the rotating still and the stationary compressor housing (see Figure 2), the compressor was not only pumping steam into the condenser, but also into the space between the rotating still and the stationary bowl. Since the walls of the stationary bowl were below the condensation temperature of the steam, product water would condense in the stationary bowl and collect there. As a result, liquid was now in a region of the DA where there was no centrifugal force, and therefore, there was no control over the liquid and no method of removing the liquid. Unchecked build-up of liquid in this area could result in drag on the still, ultimately causing a loss in performance (low production rates, high power draw) or a failure of the drive mechanism in the DA (broken drive belt). 


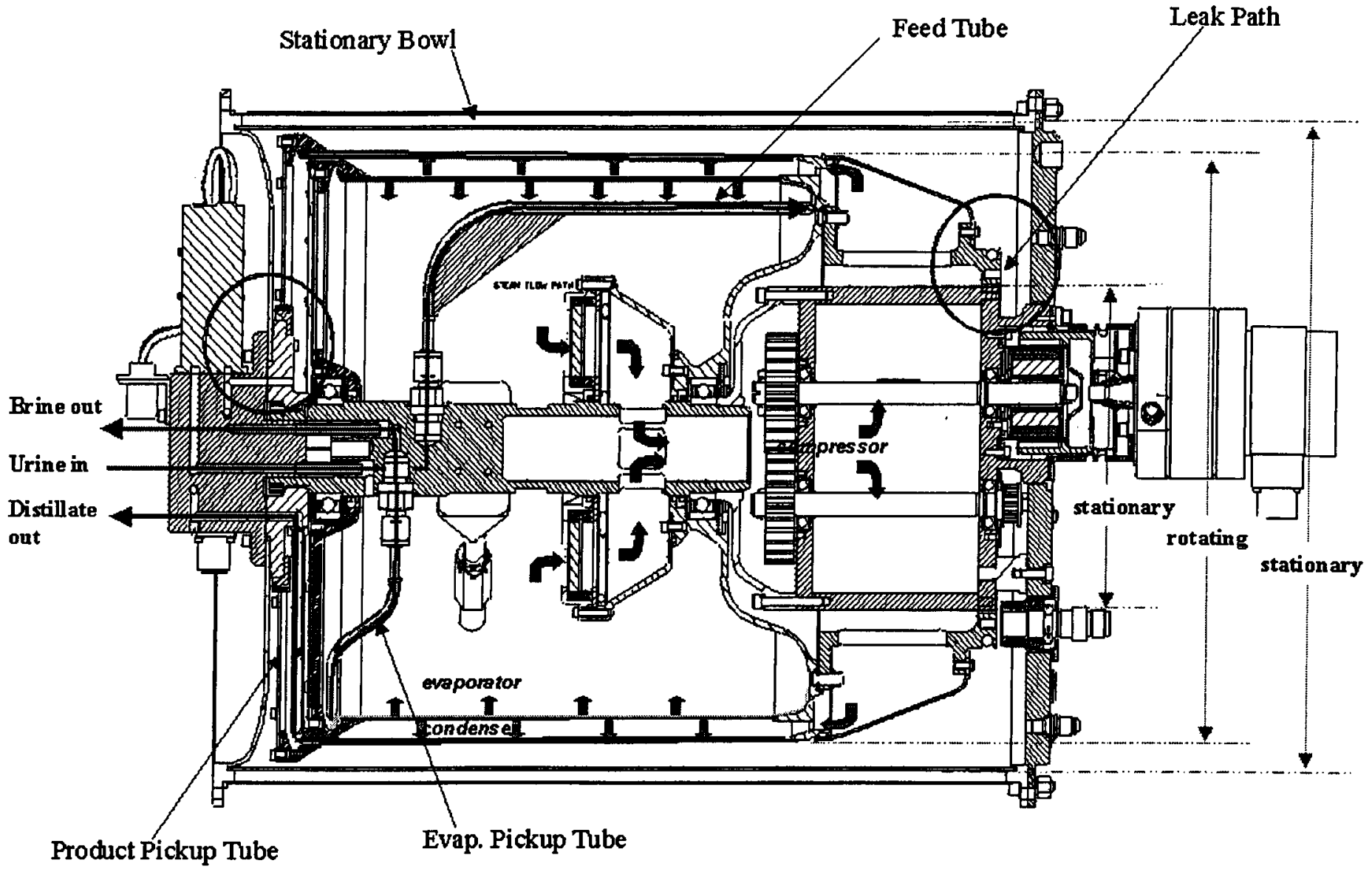

Figure 2. Cross-section of Distillation Assembly

In order to solve this problem, an extensive investigation was begun to understand how the fluid would behave in this region between the stationary bowl and the rotating still. Experts in multiple fields from rotating machinery design to microgravity fluid management were consulted. After several months of investigation, two potential solutions were selected for development to control the condensation collection in the stationary bowl: Sealing the rotating still from the stationary bowl or thermally controlling condensation of water on the stationary surfaces. Parallel development efforts were initiated. At the end of development, one of the methods was selected to incorporate into the flight design.

Sealing the rotating still from the stationary bowl was the most direct approach to preventing condensation in the bowl. The problem was in the details of the seal design. The seal itself had be tight enough so that steam would not pass through it, but the contact stresses had to be light enough so that the existing drive system for the DA would still be able to turn the still. After an extensive search, a magnetic seal design was selected. The design used the attractive force of permanent magnets to hold two halves of a dynamic face seal together. The magnetic distance and surface finish of the sealing surfaces were selected to provide the best sealing capability with the lowest contact stress. Initial development testing was not positive. Sealing was achieved but the drive belt for the still was unable to survive the additional frictional load for any significant length of time. Later in the development effort an expert in seal design was brought on board to review the design and implement modifications to increase the belt life while still providing a seal. These modifications significantly improved belt life without a loss in sealing capacity and illustrated to the design team that the dynamic sealing of the still was feasible. However, it was recognized at this point that several aspects of the DA drive system would require modification for this approach to work. Though these were considered to be feasible modifications, a development effort would have to be initiated to determine how to modify the DA to accept the magnetic seals. Since schedule had become a significant driver and success was being achieved in the thermal approach, the concept was dropped from consideration as the primary solution for controlling condensation in the stationary bowl. 
The thermal control approach is also simple in principle but not as direct as sealing the still from the stationary bowl. In this concept, condensation is prevented from occurring by controlling the stationary bowl wall temperature above the condensation temperature, thereby eliminating condensate collection in the stationary bowl. To achieve this control, heaters were wrapped around the stationary bowl and end-plates. Parametric testing was performed using a flight-like development unit to determine the sensitivities this method of control would have to several variables of the DA and fluid being processed. As a result of this testing it was shown that condensation could be controlled by external heaters, however, the success or failure of the heaters to control condensation at relatively low temperatures of $43.3-54.4\left(110-130^{\circ} \mathrm{F}\right)$ is dictated by the pressure in condenser/stationary bowl. This pressure is affected by the amount of non-condensable gas dissolved or free in the urine feed to the DA, the PCPA capacity to remove non-condensable gases from the condenser, and the compressor capacity. Through the parametric testing, allowable free and dissolved noncondensable gas levels, compressor performance modifications, and PCPA duty cycle modifications were determined that would allow the external heaters to maintain control of the condensation in the stationary bowl. These modifications have been implemented and tested in the flight-like development unit and have been shown to successfully control the condensate. As a result of this development effort, thermal control was selected as the design approach and has been implemented into the UPA flight design.

It should be noted that after the completion of this development testing and implementation of the thermal control approach into the flight design, it was determined that the dissolved gas quantities in the urine were greater than previously anticipated. Since these dissolved and free gas quantities directly affect the ability of the heaters to control condensation, a significant reduction in the free gas interface requirement (from $5 \%$ to $0.25 \%$ by volume) of the delivered urine is required to off-set the higher than expected dissolved gas in the urine. This change in interface requirements poses a technical challenge to the proposed concept for the U.S. Waste Collection System (WCS) that would have to be addressed should the WCS become an active project in the future. This required decrease in free gas will not effect the ability of the UPA to process Russian collected urine, since their separator delivers urine at close to $0 \%$ free gas.

UPA ACOUSTICS/MICROGRAVITY DISTURBANCE Throughout the long development history of the VCD technology, the main focus was on performance requirements related to production rate, power consumption, volume, and product water quality. Very little effort was given to insuring that the hardware performed its function without violating acoustic and microgravity disturbance requirements. The first experience with meeting an acoustic requirement with the VCD technology came when the VCD Flight Experiment was manifested aboard SPACEHAB. It was quickly discovered that this hardware violated the SPACEHAB acoustics requirements. A significant effort was put forth to quiet the VCD FE through acoustic foam and wraps. Though this showed some success, modification to the operational profile and a waiver were ultimately required to be able to operate the VCD FE on orbit. As a result of this experience, a significant development effort was kicked off to reduce the noise (both acoustic and low frequency) generated by the UPA.

For both the acoustics noise and microgravity environment disturbing low frequency noise, the approach was two-fold: reduce or eliminated the noise generated by the equipment, and/or prevent the noise from propagating through structure and air by isolating the equipment. For acoustics, several experts in acoustics noise generation and isolation were consulted in order to determine the source of the noise and the method for reducing or eliminating it. The noise contributors in the UPA are the two pumps (PCPA and the FCPA) and the DA, with the major acoustic noise contributor being the DA. A review of the DA design was performed and several improvements were recommended and implemented to reduce the level of acoustic noise generated. Design modifications were made to improve bearing and gear alignment and balance, either through design changes or assembly procedure modifications. In addition to these changes, the FCPA, PCPA, and DA mounting was changed to incorporate an isolator to prevent acoustic noise from propagating through the rack structure. Each noise generator was also packed in acoustic foam, and the DA was also wrapped with Bisco wrap to reduce air-born noise. Finally, the rack in which the UPA is installed is packed with acoustic foam and closed out with an acoustically designed faceplate to further contain any noise generated by the UPA. The mounting isolators, acoustic foam, wrap and rack faceplate design have been development tested. Analysis using the results from these tests indicate that the flight design should show a significant improvement from the original design baseline, and should actually be within specification for all of the frequency requirements except 250 and $500 \mathrm{hz}$ (see Figure 3). Since this analysis does not include improvements to noise generation that could be realized due to better alignment of gears and bearings and since 250 and $500 \mathrm{hz}$ frequencies are related to the gear and bearing harmonics, there is still potential for the UPA to meet all frequency requirements for acoustic noise once the final flight hardware is assembled and tested. 


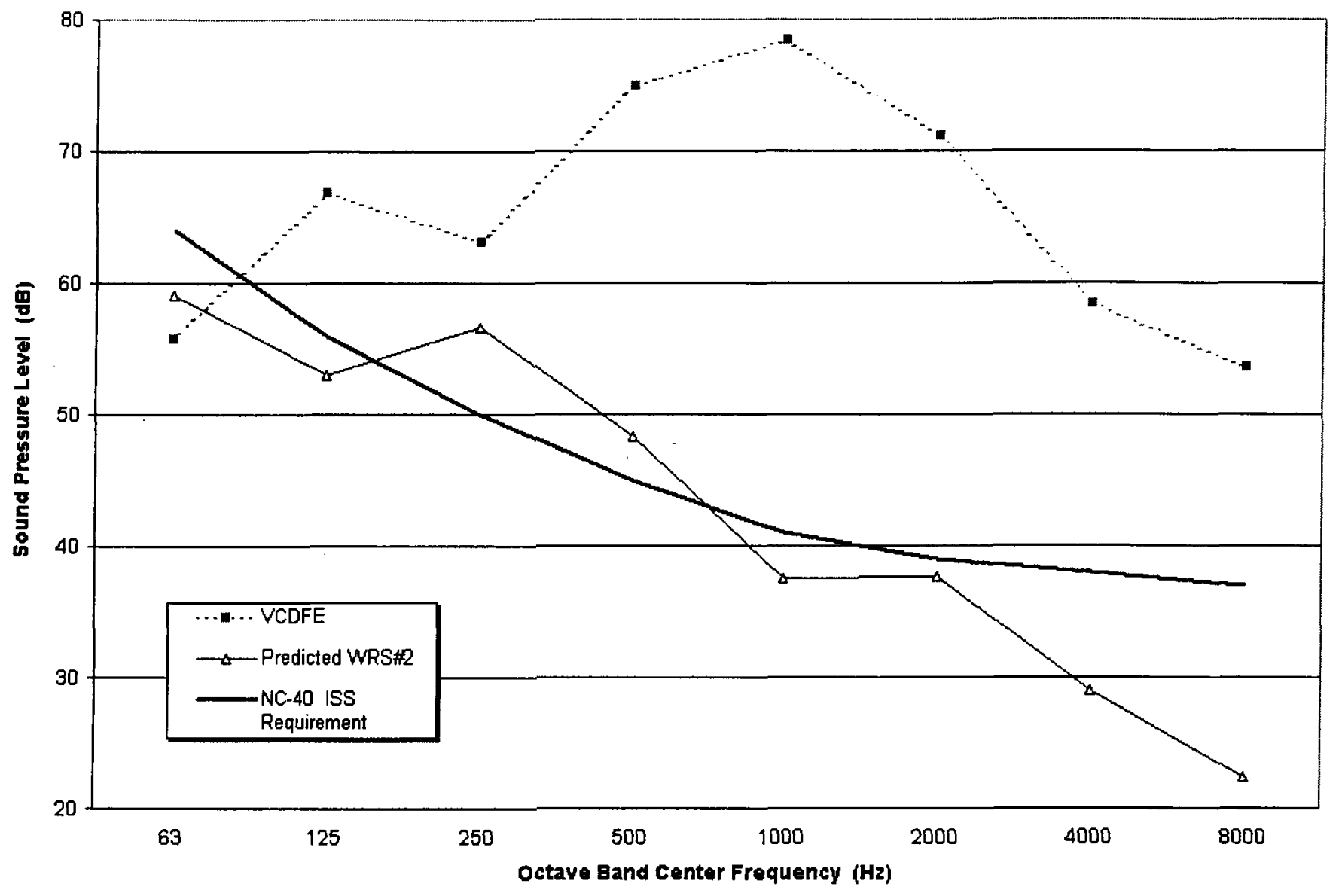

Figure 3. Predicted UPA Acoustic Performance in Water Rack \# 2 on the ISS

From a microgravity disturbance standpoint, testing has been done with the FCPA, PCPA and DA to determine the microgravity environment impact from the UPA. Initial testing showed the UPA as a bad actor in disturbing the microgravity environment on the ISS. Follow on testing with higher fidelity test configurations and UPA hardware have shown that the impact was not as great as first indicated, but was still considered a significant problem that would require design modifications to rectify it. The approach taken was to isolate the UPA hardware using passive isolator material to prevent propagation of the low frequency noise throughout the ISS. Based on test data of isolator material with the DA and low frequency noise data from the DA, FCPA, and PCPA, it has been demonstrated that the impact can be reduced by these structural isolators, however, the final analysis will not be completed until the FCPA and PCPA flight units are built and tested. At that time, it will be known whether the UPA will meet the microgravity requirements of the ISS.

UPA MICROGRAVITY PERFORMANCE - Key aspects of the UPA design have been verified and significant improvements made throughout the ground-based development history. However, an important element lacking from previous subsystem development efforts was flight-testing. Consequently, the demonstration and validation of the VCD technology and the investigation of subsystem performance in microgravity were the primary goals of the VCD FE and KC-135 experiments.

The KC-135 microgravity simulator provides short periods of microgravity (20-25 seconds). A microgravity experiment was designed and performed on the KC-135 to be able to observe what happened to water when it collected in the stationary bowl. As a secondary objective, observations were made related to the liquid level sensor fluid dynamics and the fluid flow characteristics during start and stop cycles. These experiments were designed to give some qualitative insight through visual observation of what kind of fluid behavior could be expected in microgravity. After observing the experiments realtime as well as reviewing the video and experiment log, the following conclusions were made about the UPA fluid flow characteristics:

1. Fluid which collects in the stationary bowl can be anywhere in the bowl. Only when it contacts 
both the rotating still and the stationary bowl wall does it move in a non-random way. When this occurs, the fluid is effectively pumped toward the drive mechanism, resulting in drive belt slippage. Based on these observations, water collecting in the stationary bowl was validated as a concern.

2. When excessive amounts of water were flowed into the evaporator, the water did contact the liquid level sensor as is intended. When the excessive water passes the sensor, the sensor tip is cleared by the rotational force of the bowl. From these observations, the sensor design was considered to be microgravity insensitive.

3. The concern of free floating water droplets in the evaporator after an uncontrolled stop was not observed. The water adhered to the wall of the evaporator during starts and stops.

It should be noted that the KC-135 experiment was only able to provide a qualitative assessment of how the UPA would operate on orbit. The short duration of the microgravity environment made it very difficult to observe the fluid characteristic. The final word on microgravity performance would be determined by successful operation of the VCDFE.

The VCDFE was a flight experiment aboard the Space Shuttle Columbia during the STS-107 mission. The VCDFE was a full-scale developmental version of the ISS UPA and was designed to test some of the potential micro-gravity issues with the design. Specifically, the VCDFE objectives were as follows:

1. Verify Integrated Nominal Operation in Microgravity

2. Characterize Wastewater droplet/Film Behavior during Starts and Stops

3. Confirm Gas/Liquid Separator Performance
The concept of the experiment was to run the VCDFE on the ground prior to flight and post flight and compare the flight (i.e microgravity) results to the ground to see if there were any adverse effects on the VCD technology due to microgravity. The purpose of the post flight test is so the test conditions during flight could be matched exactly for comparison to microgravity performance. Sensor data was logged and stored every second on an on-board computer as well as down linked to the ground when the signal was available. Water samples were collected during each run to verify water quality and production rate. Post flight inspections and check out as well as sampling of the brine tank would be done to see how well the system performed.

Unfortunately, when STS-107 was lost on its return to earth, so was the VCDFE hardware and a large portion of the data needed to fully evaluate the VCD technology's performance in microgravity. However, between the limited downlink data received and the crew observations that were voiced down during the mission, the VCDFE appeared to perform excellently in microgravity. The following is a brief summary of the preliminary analysis of the flight data. A final analysis is pending and will be reported in the future.

INTEGRATED OPERATION - Observations from the crew and completion of sample draws on-orbit proved that the VCDFE produced water. However, the rate cannot be confirmed since there are no actual sample volumes. Using times measured by the crew and assuming consistent sample volumes, the production rate was between 4 and $6 \mathrm{lb} / \mathrm{hr}$, which is significantly higher than the requirement.

No science was obtained from the samples as to the quality of water. The crew indicated that it was clear with no particulates, but the wastewater was also clear, so that is not conclusive. Conductivity sensors indicated the water was well within acceptable range, but this cannot be confirmed without samples (see Figure 4).

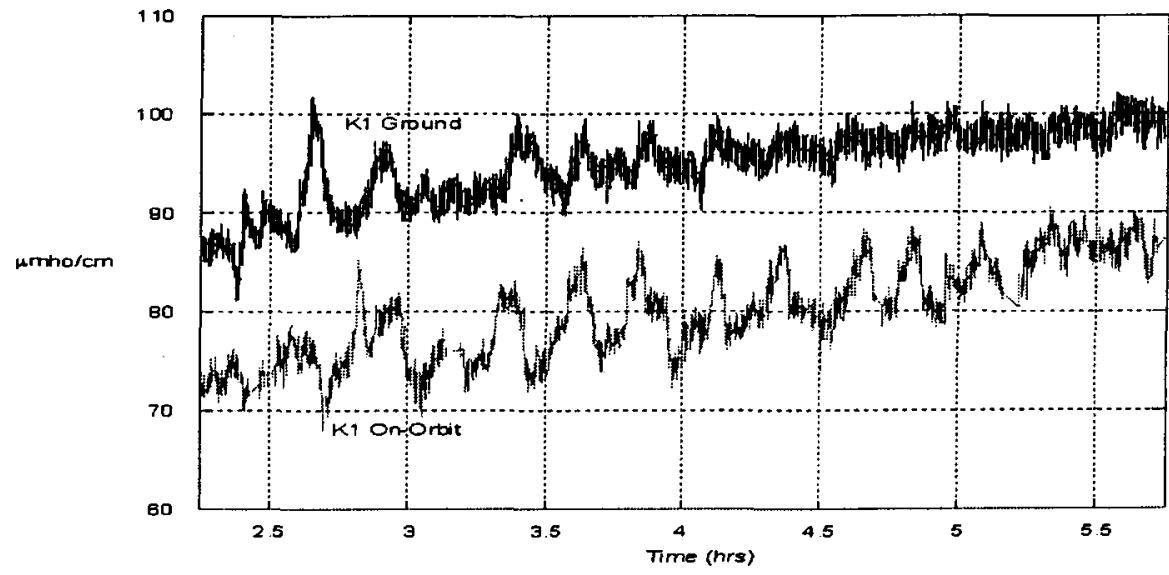

Figure 4. VCDFE Ground and Flight Comparison of Distillate Conductivity 
Downlink data gives us a good idea that the dynamic response of the VCDFE looked almost identical to ground data with one exception, the liquid level sensor indicated the evaporator was flooded beginning 30 minutes into the first run and never cleared (even after drydown was completed). This could be an indication that the fluid dynamics are different in micro-gravity (resulting in water never clearing the sensor head even though the evaporator is not flooded), the sensor failed or there actually was more liquid in the evaporator than thought. The fact that no other sensors indicated a flooded condition (still speed and current draw were nominal) and the observation made during the KC-135 experiment would seem to eliminate flooding of the evaporator. Only post-flight inspection of the sensor and a mass balance of the system water could have definitively answered this question.

\section{CHARACTERIZATION OF WASTEWATER} DROPLET/FILM BEHAVIOR - This objective was achieved by starting and stopping the VCDFE without drying out the evaporator. The objective was to see if the system responded differently because of free water that could potentially impact the compressor performance or product water quality. Multiple starts and stops were run during the mission and none of the downlink data indicated that either the product water quality or compressor performance was affected. This was considered one of the major concerns of the VCD technology, and the existing flight data indicates that uncontrolled starts and stops due to power loss should not effect the UPA operation. This also appears to confirm the observations made during the KC-135 experiment.

GAS/LIQUID SEPARATOR PERFORMANCE - Based on the flight downlink data, the gas/liquid separator performance appeared to match ground data for a properly performing membrane separator.

In summary, the VCDFE and KC-135 data that has been analyzed to date indicated that the technology is well suited for microgravity operation. With the exception of the liquid level sensor anomaly during flight, everything compared well to ground data. Since the real reason for the high liquid level reading will never be known, design engineers are presently trying to identify all possible causes of a high liquid level reading and develop modification to the flight hardware (if any) that would mitigate the chance for a high reading to occur during flight operations.

UPA PROCESSING RUSSIAN PRE-TREATED URINE As was alluded to in an earlier section, due to ISS budget issues, the WCS that feeds the UPA pre-treated urine was put on hold. As a result, the UPA project was asked by the ISS program to determine the feasibility of processing Russian pre-treated urine.

Table 1 lists the major differences between Russian pretreated urine and U.S. pretreated urine. The major difference results from the quantity and type of pretreatment chemicals used in the U.S. verses Russian pre-treated urine. To assess the potential impacts, the problem was divided into 3 major areas: UPA performance, UPA materials compatibility, UPA design features for safety.

Table 1. Comparison of U.S. and Russian Pretreated Urine

\begin{tabular}{|l|l|l|}
\hline Parameter & $\begin{array}{l}\text { U.S. Pretreated } \\
\text { Urine }\end{array}$ & $\begin{array}{l}\text { Russian } \\
\text { Pretreated } \\
\text { Urine }\end{array}$ \\
\hline $\begin{array}{l}\text { Pre-treatment } \\
\text { Chemicals }\end{array}$ & $\begin{array}{l}\text { Oxone/Sulfuric } \\
\text { Acid/potassium } \\
\text { benzoate }\end{array}$ & $\begin{array}{l}\text { Chromium } \\
\text { Trioxide/Sulfuric } \\
\text { Acid }\end{array}$ \\
\hline PH & $2.4-2.6$ & $1.3-2.0$ \\
\hline$\%$ Free Gas & $5 \%$ & $0 \%(1)$ \\
\hline
\end{tabular}

Note 1 - per conversations with Russian designers

In order to address the performance impacts of the Russian pretreated urine on the UPA, the development UPA has been run for over one hundred hours. Data from this testing (both operational and product water quality data) was then compared to the operational data of the development UPA while processing U.S. pretreated urine. Operational data (current draw, pressures, temperature) and water quality data all compared favorably for using the UPA to process Russian pretreated urine. Table 2 shows a comparison of the water quality data that indicates that the Russian pretreatment may actually improve water quality. Based on these results, it was determined that the Russian pretreatment should have no adverse effects on the UPA performance.

Table 2. Comparison of U.S. and Russian Pretreated Urine Distillate Water Quality

\begin{tabular}{|l|l|l|}
\hline Parameter & $\begin{array}{l}\text { U.S Pretreated } \\
\text { Urine Distillate }\end{array}$ & $\begin{array}{l}\text { Russian } \\
\text { Pretreated } \\
\text { Urine Distillate }\end{array}$ \\
\hline TOC & $150 \mathrm{mg} /$ liter & $55 \mathrm{mg} /$ liter \\
\hline TIC & No Data & $1.1 \mathrm{mg} /$ liter \\
\hline Conductivity & 137 umhos $/ \mathrm{cm}$ & 150 umhos $/ \mathrm{cm}$ \\
\hline PH & 3.2 & 3.15 \\
\hline
\end{tabular}

A concern was raised about the materials compatibility of the UPA hardware to the Russian pre-treated urine. An engineering assessment of the materials in the UPA and the Russian composition did not identify any known 
issues with the materials compatibility. However, in order to definitively answer this question, materials compatibility testing has begun with Russian pretreated urine and concentrated Russian pretreated urine (urine brine). The testing is approximately $1 / 3$ complete with no indication of problems to date.

The final concern relates to the containment of the pretreated urine and brine by the UPA. The UPA was design to contain toxicity level 1 fluid. The toxicity rating was mainly due to the $\mathrm{pH}$ level of the U.S. pretreated urine. Toxicity level 1 requires 2 levels of containment for which the UPA is presently designed. The most significant design features associated with 2 containment levels are dual o-ring seals. The toxicity of the Russian pretreated urine is at level 2. This requires 3 levels of containment and fracture critical requirements on storage tanks. Since the UPA design is complete and the hardware is either built or is in the process of being built, changing the design to incorporate 30 -rings at every fluid mechanical joint or implementing fracture critical requirements on the tanks would be extremely cost and schedule prohibitive. Therefore, rationale has been generated based on the existing design, materials compatibility test results, and operational modifications that would allow the UPA to process Russian pre-treated urine under a waiver. The initial rationale has been presented to the ISS Safety Review Panel (SRP). Final approval of the waiver is on hold pending the completion of the materials compatibility testing.

\section{SUMMARY}

The development of the UPA technology has been ongoing for decades. The initial development effort focused on designing a system that could actually achieve the function of distilling urine in a microgravity environment. Once this was achieved, the development effort for the UPA was considered completed. Only when it came time to do final design on the flight hardware were these additional development efforts started. In most cases, it was a technical issue that was not addressed either through oversight (i.e. condensate in the stationary bowl, acoustics/microgravity disturbance) or because requirements changed late in the project (i.e. processing Russian pretreated urine). Microgravity performance verification was always recognized as necessary, but because of the cost and schedule delays, was only completed late in the flight program. These efforts have been successful and, though the timing has not been optimal, they have supported the flight system design and manufacturing effort. Though in any program areas will be missed that will require development, the lesson learned from this experience is that during the initial development of a technology, resources should not only be put toward making the technology achieve the functional performance requirements, but effort should be spent in insuring the technology will also meet environmental and safety requirements. These are common requirements to all space flight technologies that should be considered early in the development of a system to insure that the technology is able to perform its function in the space flight environment.

\section{ACKNOWLEDGEMENTS}

The authors would like to thank the many designers and technical experts that participated in all of these efforts so that the flight design of the UPA could be realized. Specifically, we would like to thank David Long, Rex Graves, Tom Williams, and Keith Parrish for their tireless support of all aspects of the development work described above. We would like to thank Brian Mitchell for providing his mechanical design and seal design expertise, Doug Counter and Ed O'Connor for rescuing us from our acoustics problem, and Janice Houston for making sure we do not disturb the ISS microgravity environment.

Finally, we would like to give a special thanks to the crew of STS-107 Columbia. Through their sacrifice we gained invaluable knowledge of the VCD technology and how it operates in microgravity.

\section{REFERENCES}

1. Carrasquillo, R.L., "Status of the Node 3 Regenerative ECLSS Water Recovery and Oxygen Generation Systems", SAE \# 2003-01-2590, presented at the $33^{\text {rd }}$ International Conference on Environmental Systems, Vancouver, Canada July , 2003 


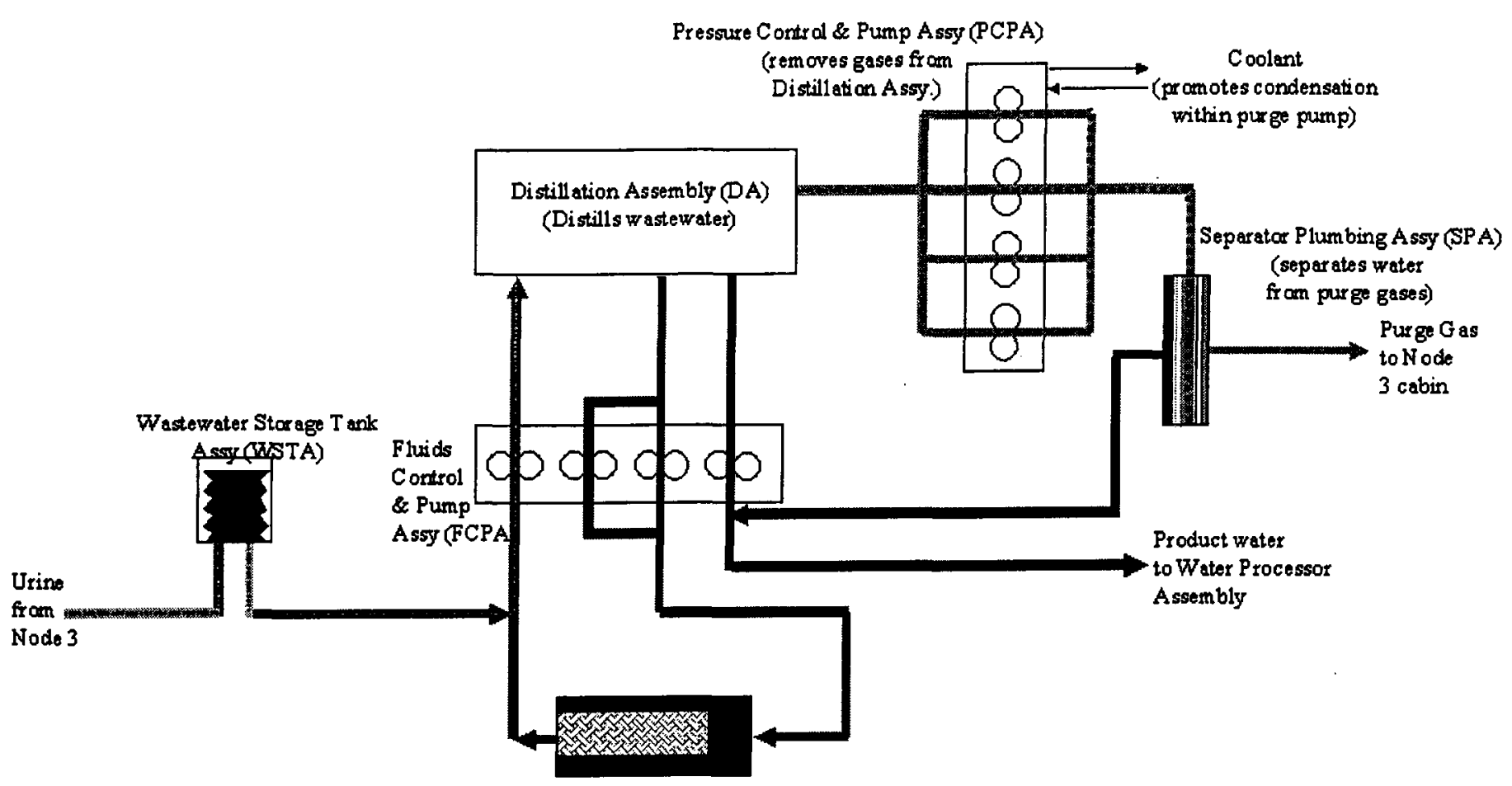

Recycle Filter $T$ ank Assy. (RFT A)

(accumulates \& stores

brine for disposal) 


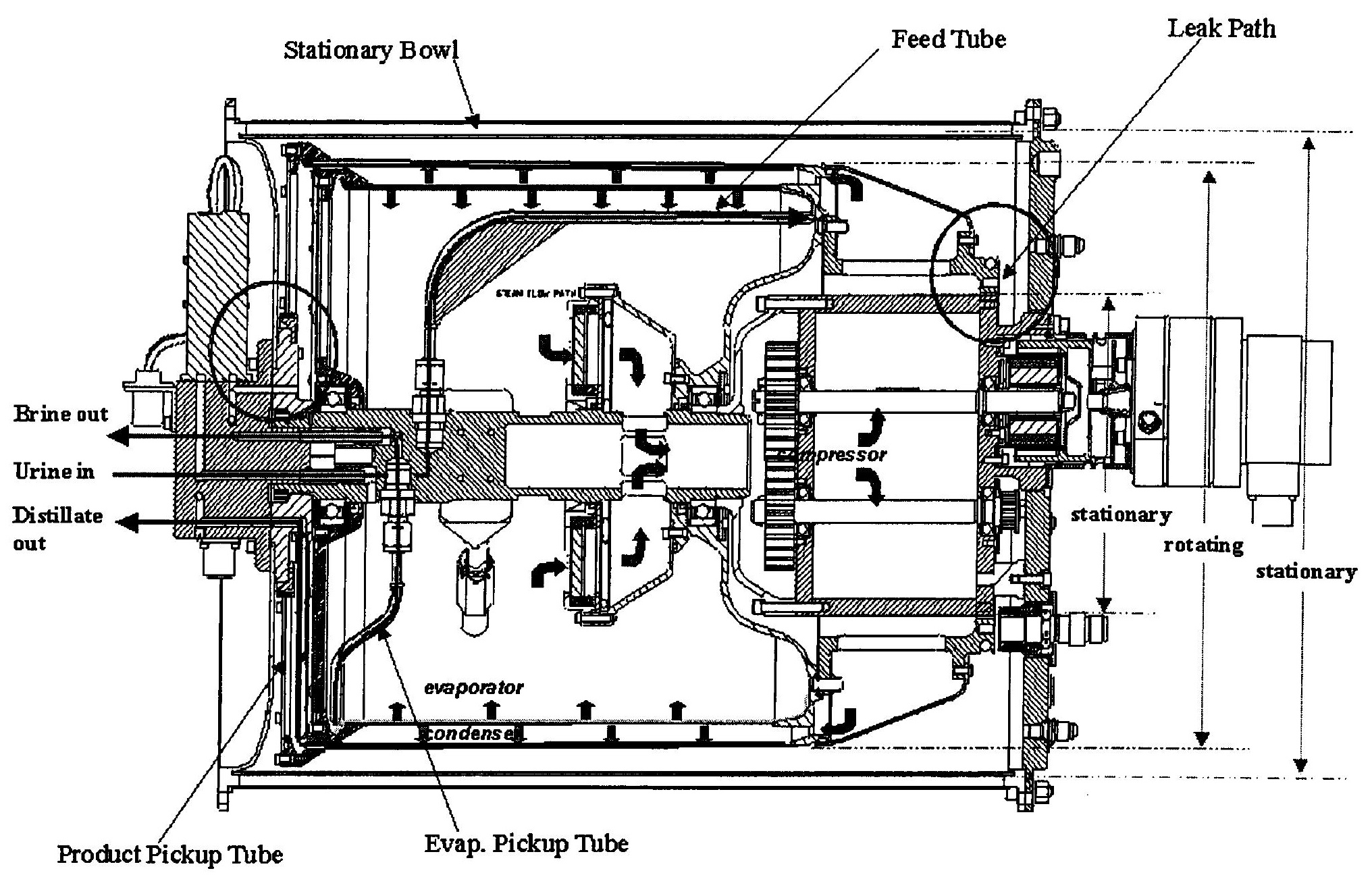




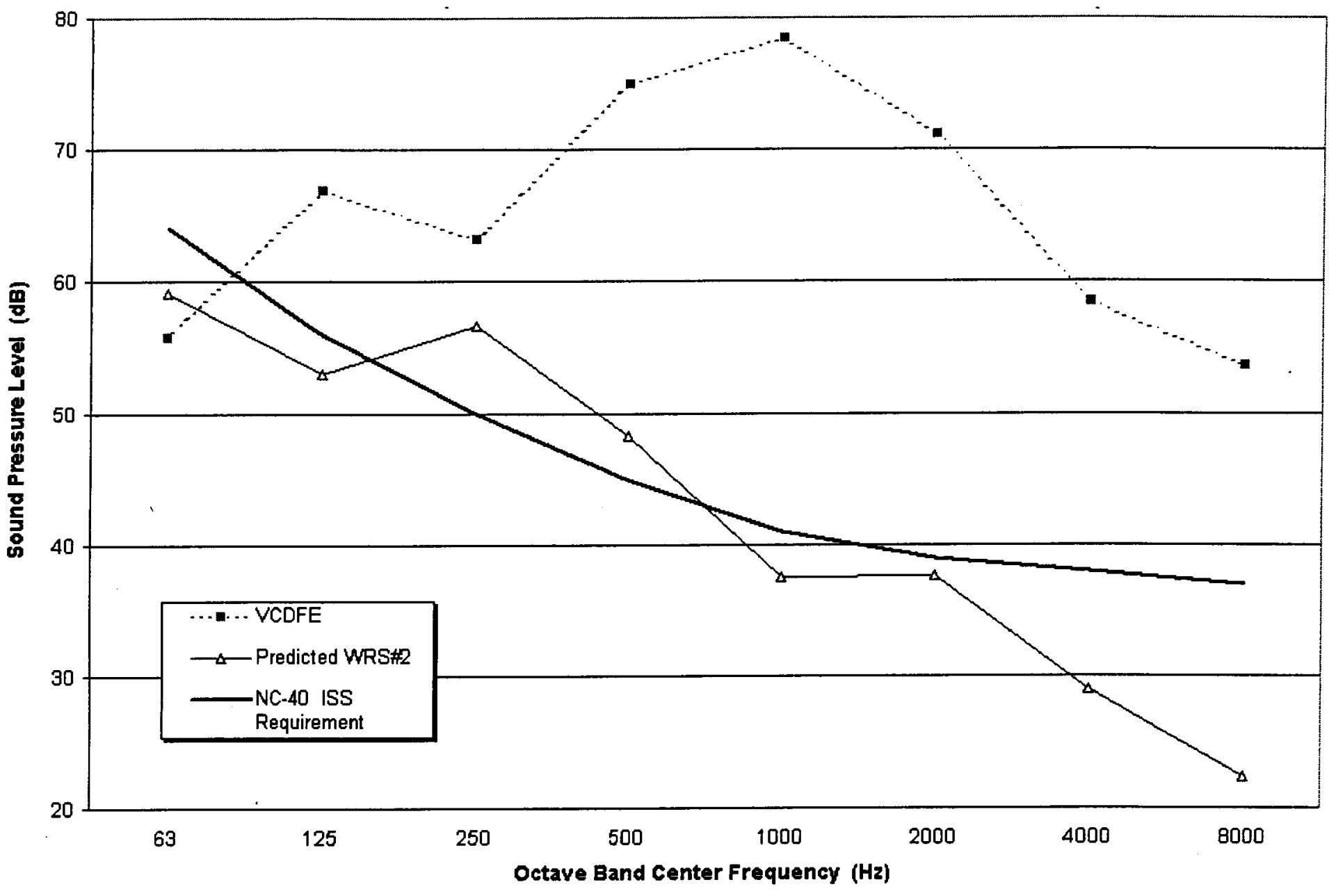




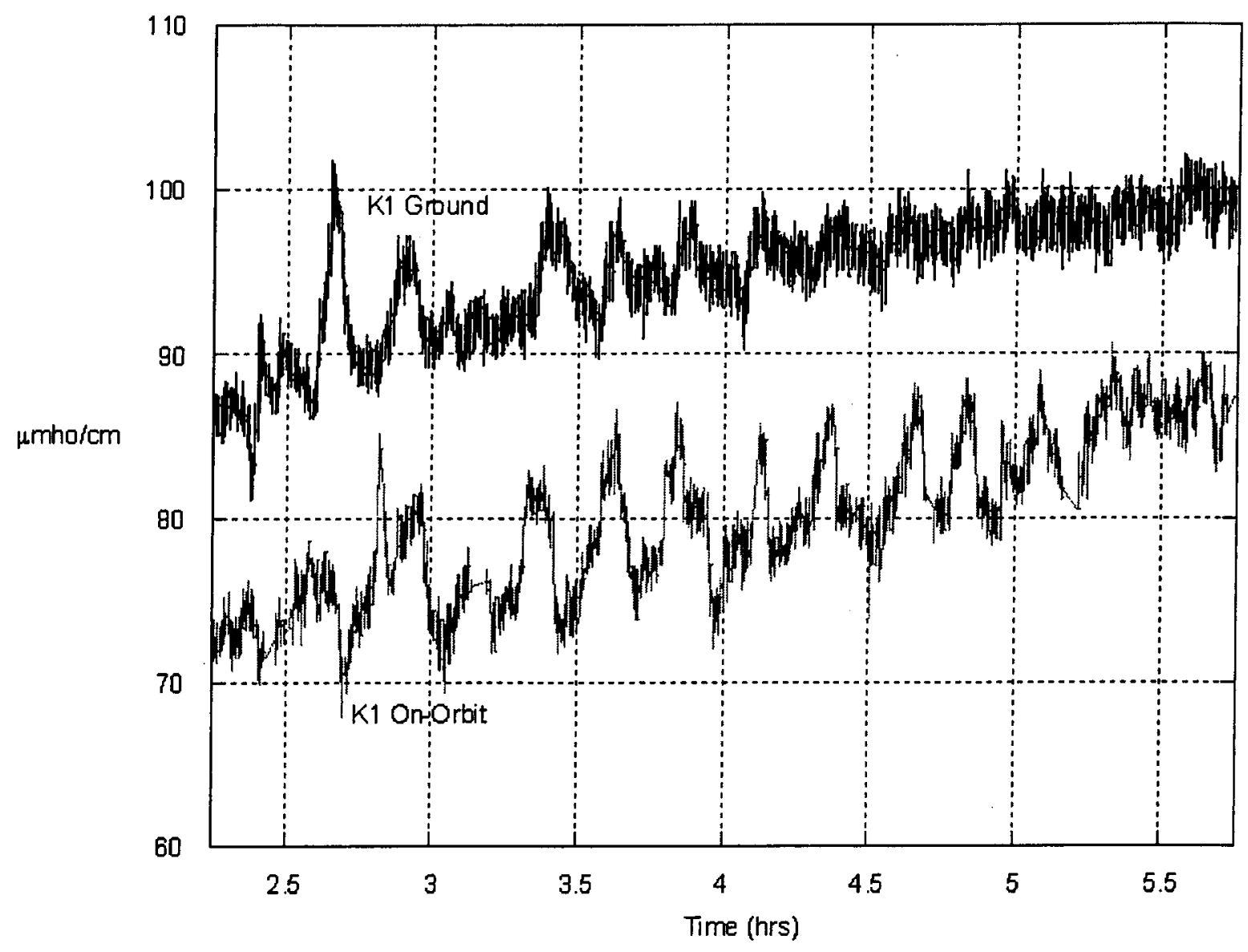




\section{IMPORTANT CONFERENCE REGISTRATION INFORMATION}

ADVANCE REGISTRATION SAVES TIME AND MONEY! To register for 33rd International Conference on Environmental Systems, go to www.sae.org/ices prior to June13, 2003. Please enter the source code of 030825.

\section{Here are some ways to register - which one best fits you?}

\section{SAE Members}

SAE Members attend this conference at a discounted price. If you are a current SAE member you do need to pre-register. You can register online or call $724 / 776-4970$. You receive the largest discount if yo register before June 13, 2003

\section{Not a Member?}

Join SAE by June 13, 2003 and take advantage of the many benefits of SAE membership...and register for this conference at Member pricel Plus, receive a FREE subscription to Aerospace Engineering or Automotive Engineering Intemationa/ magazine, 20\% discount on all SAE Bookstore products (technical papers, standards, and books (in electronic and print format) under $\$ 500$, Network with industry leaders and corporate officials at SAE meetings and local Section activities, and much more as part of your SAE membership! Tojoin SAE before the pre-registration date, June 13, 2003, visit www.sae.org, go to the Membership button on the top menu bar, and select "Join SAE Now!" Complete the online membership application and save $\$ 10.00$ ! Plus, receive immediate access to all the benefits membership has to offer. Your membership will be complimentary for 1 year.

\section{Non-member}

Registrants

Individuals paying the Non-member Registrant or the Non-member Student fees will receive a FREE membership in SAE for 1 year. Visit www.sae.org, go to the Membership button on the top menu bar, and select Membership Forms - print out the pdf application and submit application with your pre-registration form or drop it off at the registration desk when you pay the non-member registration fee on-site.

\section{Paid Full Conference Attendees}

Individuals paying the full conference fee are entitled to attend the technical sessions, coffee breaks, receptions, and receive one ticket to the banquet and a CD-ROM containing the ICES technical papers.

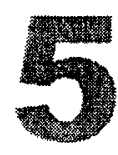

\section{One-Day Attendees}

Individuals registering for one-day may attend technical sessions for that day only. Registration does not include the banquet or the proceedings on CD-ROM.

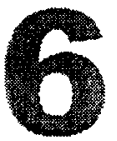

\section{Media and Students}

Individuals registering as Media or Student are entitled to attend technical sessions. Full time Student ID and Media Credentials are required. Registration does not include proceedings or a banquet ticket.

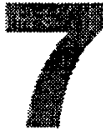

\section{Technical Program Participants}

Primary authors, session chairs \& organizers, and panelists are entitled to attend the technical sessions, FREE, on the day of their presentation. To attend on the other days, the full conference or one-day conference fee must be remitted.

Additional categories located on next page (pre-registration form)

\section{Other Registration Notes:}

- On-site registration takes place in the level two foyer of the Westin Bayshore Resort \& Marina.

- Pre-registration deadline is June 13, 2003.

- Registration will be available during the following times:

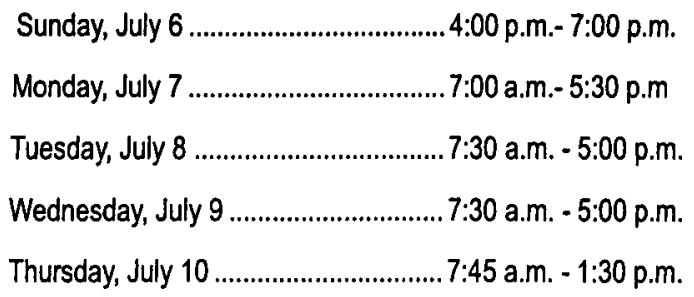

- Children under 16 years of age are not permitted to attend. 